\title{
Gestion des patients à risque de dégénérescence maculaire liée à l'âge: une stratégie canadienne
}

\section{RÉSUMÉ}

Contexte : Élaborer une stratégie consensuelle pour la gestion des patients à risque de dégénérescence maculaire liée à l'âge (DMLA) à l'intention des ophtalmologistes, optométristes et médecins du Canada.

Méthodes : L'élaboration d'une stratégie consensuelle a débuté avec la recension des textes et un examen des lignes directrices existantes. Un groupe de spécialistes de la rétine, d'ophtalmologistes et d'optométristes provenant d'un bout à l'autre du Canada a évalué cette information probante pour distiller ce qui a été appris et utiliser ces connaissances comme point de départ pour l'élaboration d'une stratégie consensuelle pour la gestion des patients à risque de DMLA.

Résultats : Le groupe d'experts a élaboré une série de recommandations à l'intention des fournisseurs de soins oculovisuels (p. ex., ophtalmologistes, optométristes) et médecins du Canada pour l'adoption d'une stratégie préventive dans le cas des patients à risque de DMLA.

Interprétation : La présente stratégie consensuelle est une ligne directrice pratique qui peut être adoptée dans un bureau pour gérer les patients à risque de DMLA et pour informer les patients qui ont des questions ou des préoccupations relatives à la DMLA.

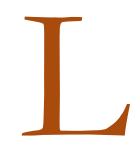

a dégénérescence maculaire liée à l'âge (DMLA) est une maladie dégénérative incurable et la cause principale de la cécité chez les adultes de plus de 50 ans dans les pays développés ${ }^{1}$. Elle a des conséquences socioéconomiques appréciables qui continueront de prendre de l'ampleur avec le vieillissement de notre population, à moins de trouver une façon de prévenir la DMLA ou d'arrêter sa progression.

Il y a deux types de DMLA. La DMLA néovasculaire, aussi appelée « exsudative » et « humide », est caractérisée par l'apparition de membranes néovasculaires choroïdiennes, d'hémorragie et/ou de fluide sousrétinien, puis de fibrose, au stade avancé. La forme la plus courante, sèche et atrophique, représente environ $85 \%$ des cas de DMLA. Elle est caractérisée par des drusens et/ou des irrégularités épithéliales pigmentaires de la rétine, qui progressent souvent aux régions atrophiées $^{2}$. Un faible pourcentage des cas de DMLA sèche évoluent en DMLA néovasculaire ${ }^{2}$.

L'histoire naturelle de la DMLA est complexe et mal comprise, bien que divers facteurs de risque aient été déterminés. La DMLA est in- curable mais les récentes découvertes permettent de plus en plus de la gérer. En outre, un aperçu de la prévention et des facteurs de risque a aidé à l'élaboration d'autres stratégies importantes de gestion.

À Toronto en 2007, un groupe d'experts formé d'ophtalmologistes et d'optométristes du Canada s'est réuni pour atteindre les objectifs suivants :

- Comprendre les pratiques exemplaires actuelles en matière de gestion des patients à risque de DMLA.

- Comprendre les défis actuels en matière d'aide aux patients à risque afin de réduire l'incidence de cette maladie débilitante et de prévenir sa progression aux stades avancés.

- Élaborer des lignes directrices pratiques pour aider à prévenir la progression de la DMLA chez les patients à risque.

Le but des présentations et des discussions subséquentes à la réunion était de présenter un contexte, un point de vue et de l'information tirée de la documentation existante et de la pratique clinique pour servir de base à la définition d'une straté- 
gie consensuelle pour la gestion des personnes à risque de DMLA.

\section{Incidence et prévalence de la DMLA}

On ne dispose pas de chiffres précis sur la prévalence de la DMLA au Canada; toutefois, il n'est pas déraisonnable d'extrapoler à partir d'une importante initiative américaine : the Beaver Dam Eye Study. Durant la première étude (1988-1990), Klein et $\mathrm{al}^{3}$ ont déterminé qu'il existait des signes de maculopathie liée à l'âge chez 8,5\% des personnes de 43 à 54 ans, et que ces signes étaient plus courants chez les personnes de 75 ans et plus (36,8\%). Dans l'étude suivante (2003-2005), Klein et $\mathrm{al}^{4}$ ont rapporté que l'incidence cumulative sur 15 ans de la DMLA au stade précoce était de 14,3\% chez les adultes de moins de 43 ans; alors que l'incidence de la DMLA au stade avancé était de 3,1\%. Parmi les personnes de 75 ans et plus, l'incidence cumulative sur 15 ans de la DMLA au stade avancé correspondait à $8 \%$. L'extrapolation des constatations initiales de la Beaver Eye Dam Study à la population canadienne permet d'établir que presque 2,2 millions de Canadiens pourraient souffrir de DMLA à un stade plus ou moins avancé (tableau 1).

Étant donné la baisse du taux de natalité et la prolongation de l'espérance de vie, la proportion d'adultes canadiens plus âgés s'accroît plus rapidement que celle des autres groupes d'âge ${ }^{5}$. En 2005, on prévoyait que le nombre de Canadiens âgés (de plus de 65 ans) s'accroîtrait, et passerait de 4,2 millions à 9,2 millions en 2036, doublant presque le pourcentage de personnes âgées dans la population canadienne, en passant de 13,2\% à $24,5 \%{ }^{5}$

\section{Le fardeau de la maldie}

Brown et $\mathrm{al}^{6}$ ont rapporté que même un cas léger de DMLA (20/20 à 20/40 dans l'œil qui voit le mieux) diminue la qualité de vie de $17 \%$ chez le patient moyen, des conséquences semblables à celles d'une angine cardiaque modérée ou du VIH symptomatique. La DMLA modérée (20/50 à 20/100 dans l'œil qui voit le mieux) entraîne une diminution de $32 \%$ de la qualité de vie, des conséquences semblables à celles d'une angine cardiaque grave ou d'une fracture de la hanche. La DMLA grave $(\leq 20 / 200$ dans l'œil qui voit le mieux) diminue la qualité de vie de $53 \%$, ce qui correspond à des conséquences plus importantes que celles de la dialyse rénale. Les personnes souffrant de DMLA très grave $(\leq 20 / 800$ dans l'œil qui voit le mieux) ont déclaré une diminution de $60 \%$ de leur qualité de vie, des conséquences semblables à celles du cancer de la prostate au stade terminal ou d'un accident vasculaire cérébral invalidant. Il convient de souligner que Brown et $\mathrm{al}^{6}$ ont affirmé que les ophtalmologistes sousestimaient énormément la qualité de vie des patients souffrant de DMLA modérée à grave.

Ces auteurs ont aussi rapporté que les formes humide et sèche de la DMLA ont des retombées négatives annuelles qui se chiffrent à approxi- mativement 30 milliards de dollars américains sur le produit intérieur brut des États-Unis ${ }^{6}$. Une analyse économique plus récente a estimé le coût indirect de la DMLA humide à 2,5 milliards de dollars américains par année en perte de productivité 7 . Pour la forme sèche de la DMLA, les coûts indirects se chiffrent à 24,4 milliards de dollars américains ${ }^{7}$.

\section{Pathogénèse de la DMLA}

La pathogenèse de la DMLA est multifactorielle et elle dépend de facteurs génétiques et environnementaux. Elle est associée à des interactions de plusieurs processus biologiques, incluant le stress oxydatif et l'inflammation, qui entraînent une dysfonction de l'épithélium pigmentaire de la rétine (EPR), de la membrane de Bruch et du complexe photorécepteur. L'activation de ces processus provoque une chaîne d'événements (figure 1), dont les manifestations constituent les caractéristiques de la DMLA $^{4}$ :

- L'apparition d'un grand nombre de petits drusens durs entre l'EPR, la membrane de Bruch et la choroïde.

- Les drusens deviennent plus gros et plus confluents ("plus mous »). Il se produit des anomalies pigmentaires (hyperpigmentation et atrophie) et la lipofuscine augmente dans l'EPR.

- Au fil du temps, l'atrophie géographique centrale de l'EPR et/ou l'angiogénèse peut suivre. 
L'analyse permettait de conclure que, lorsque considérés séparément, il n'y a pas assez d'éléments probants qui appuient le rôle des antioxydants alimentaires, incluant l'utilisation de suppléments alimentaires antioxydants, dans la prévention primaire de la DMLA au stade précoce. Les auteurs faisaient remarquer que la métaanalyse présentait une restriction, à savoir qu'ils n'avaient nullement examiné les effets des antioxydants combinés.

L'étude de Rotterdam, une étude de cohortes prospective, a permis de comparer les effets des antioxydants pris séparément et combinés. On a rapporté que les résultats pour les antioxydants pris séparément n'étaient pas aussi forts que l'effet bénéfique rapporté quand on prenait les quatre suppléments antioxydants combinés? ${ }^{9}$. Cette constatation était semblable à celle de l'étude AREDS, où l'on rapportait d'importantes réductions du risque de la progression de la DMLA avancée dans la branche des antioxydants (17\%), la branche du zinc $(21 \%)$ et la branche antioxydants et zinc (25\% après 6,3 ans et $27 \%$ dans la prolongation de 10 ans $)^{15}$.

Peutêtre que les effets bénéfiques dans les deux études sont attribuables aux effets synergiques des antioxydants pris ensemble que séparément.

Le fait qu'une grande partie de la population observée pendant l'étude travaillait dans le secteur des soins de santé, et qu'elle pourrait par conséquent ne pas être représentative de la population normale ${ }^{21}$, est une autre restriction de la métaanalyse.

\section{Les facteurs de risque}

On ne comprend pas entièrement de manière précise comment débute et progresse le processus pathologique de la DMLA mais plusieurs facteurs de risque ont été déterminés. Ils peuvent être répartis en deux groupes, les facteurs non modifiables et les facteurs modifiables.

\section{Les facteurs de risque non modifiables}

L'âge : La Beaver Dam Eye Study, entre autres, a permis d'établir que l'incidence et la gravité de la DMLA augmentent avec l'âge 4 .

La génétique : Selon des études de jumeaux et de familles, il semble y avoir de solides éléments probants appuyant l'existence d'une composante génétique dans le risque de souffrir de DMLA ${ }^{2,22}$. De récentes études sur les liens et les associations génomiques se sont concentrées sur la découverte de gènes candidats pour la DMLA. Parmi les loci d'intérêt se trouvent 1q32 (gène complément du facteur $\mathrm{H}$ ) et une région de 10q26 (LOC387715). Bien qu'on ne connaisse pas le mécanisme exact selon lequel ces polymorphismes de nucléotides simples prédisposent à la DMLA, ils semblent jouer un rôle dans les modèles de cascade inflammatoire et de stress oxydatif d'une blessure, respectivement $\mathrm{t}^{23}$.

Le sexe : Les femmes sont légèrement plus à risque de développer un ou plusieurs drusens intermédiaires, de petits drusens généralisés, ou des anomalies pigmentaires de l'EPR ${ }^{12}$.

La race : L'AREDS 3 a permis de trouver une plus haute fréquence de néovascularisation choroïdienne chez les personnes de race blanche, chez qui on avait plus fréquemment observé un ou plusieurs grands drusens $(>125 \mu \mathrm{m})$ et une grande quantité de drusens intermédiaires ${ }^{12}$. Les auteurs ont avancé l'hypothèse qu'une augmentation de mélanine dans les cellules EPR, qui agit comme un capteur de radicaux libres ou simplement comme un filtre pour les rayons ultraviolets, protège les cellules EPR et la membrane de Bruch, réduisant ainsi le risque de développer de grands drusens et de modifications pigmentaires de l'EPR et réduisant, de cette façon, le risque d'atrophie géographique, de néovascularisation choroïdienne, ou les deux ${ }^{12}$.

La couleur des yeux : Hyman et $\mathrm{al}^{24}$ ont constaté un lien entre les yeux bruns et une plus faible DMLA. Frank et $\mathrm{al}^{25}$ ont conclu qu'il y avait une plus grande prévalence de DMLA chez les personnes de race blanche qui ont un iris de couleur pâle que chez les personnes dont l'iris est de couleur plus foncée.

Les affections concomitantes : Les chercheurs de l'étude AREDS ont trouvé une corrélation entre la DMLA et les affections concomitantes, comme l'hypermétropie ${ }^{12}$, le diabète ${ }^{26}$, et des antécédents de maladie cardiovasculaire ${ }^{26}$.

\section{Les facteurs de risque modifiables}

Le tabagisme : De tous les facteurs de risque modifiables, le tabagisme est le plus important. Les études AREDS 3 et 19 ont permis de 
constater que le tabagisme était associé à des cas plus graves de DMLA (humide et sèche) ${ }^{12,20}$. On ne connait pas exactement le mécanisme de la détérioration mais les auteurs de l'étude AREDS 3 ont fait remarquer que le tabagisme diminue les niveaux d'antioxydants en circulation ${ }^{12}$. Ils ont proposé une raison vasculaire sous-jacente à la DMLA parce que le tabagisme peut détériorer les vaisseaux choroïdiens et altérer le débit sanguin choroïdien, favorisant ainsi des changements athéroscléreux et hypoxiques dans les vaisseaux choroïdiens. Le tabagisme peut entraîner directement la vasoconstriction, augmentant l'hypoxémie et le stress oxydatif $^{12}$.

Les facteurs liés au style de vie : Les facteurs liés au style de vie, comme le régime alimentaire, la forme physique, l'obésité et les facteurs socioéconomiques jouent un rôle dans la DMLA ${ }^{20}$.

L'exposition au soleil : L'exposition aux rayons solaires ultraviolets est un autre facteur de risque modifiable. Bien que la preuve du rôle pathogène de l'exposition solaire dans la DMLA soit controversée, elle ne peut être ignorée ${ }^{9,13,14}$.

\section{Consensus}

- La DMLA est une maladie plurifactorielle. Les effets relatifs des facteurs de risque environnementaux et génétiques sont inconnus.

- De nombreux facteurs de risque de DMLA sont bien connus, comme en témoignent les publications médicales.

- Il manque de données cliniques probantes pour conclure que la gestion des facteurs de risque peut prévenir la progression de la DMLA au stade précoce. Les essais contrôlés randomisés, comme l'étude AREDS, n'ont pas formulé de recommandations conclusives pour les patients au stade précoce de la DMLA (catégorie 1 ou 2).

- Les essais contrôlés randomisés, comme l'étude AREDS, ont montré que la gestion des facteurs de risque modifiables a un effet sur la DMLA au stade avancé. Chez les patients des catégories 3 et 4 de la DMLA, la norme de soins inclut la gestion des facteurs de risque modifiables.

- Malgré les données probantes provenant des essais contrôlés randomisés, bon nombre de personnes ne sont pas convaincues de l'importance de la gestion des facteurs de risque.

- Les facteurs de risque non modifiables comme l'âge, la génétique, le sexe, la race, la couleur des yeux et les affectations concomitantes, font toujours l'objet d'études.

L'importance des facteurs de risque modifiables, comme le tabagisme, la nutrition (incluant les antioxydants comme les vitamines $C$ et $\mathrm{E}$, la lutéine, la bétacarotène, la zéaxanthine et le zinc), les facteurs liés au style de vie et l'exposition au soleil, doit être renforcée pour sensibiliser les patients.

- Le rôle éducatif des professionnels des soins oculovisuels en ce qui a trait au renforcement de l'importance des facteurs de risque modifiables est fondamental.

\section{Le problème alimentaire}

Le Guide alimentaire canadien de 2007 recommande sept (7) portions de fruits et légumes quotidiennement pour les adultes de plus de 51 ans $^{27}$. Les fruits et légumes sont d'excellentes sources naturelles de lutéine et autres antioxydants; cependant, une grande proportion de Canadiens de plus de 50 ans ne consomment pas les rations quotidiennes recommandées (tableau 2$)^{28}$. Les suppléments d'antioxydants peuvent être la réponse, comme le laissent entendre les études Rotterdam Eye Study et AREDS $12,15,16$.

\section{Consensus}

- On prévoit que l'incidence de la DMLA augmentera considérablement dans les années à venir en raison du vieillissement de la population du Canada.

- Les mauvaises habitudes alimentaires des Canadiens plus âgés pourraient aggraver la progression prévue de la DMLA en raison du vieillissement.

- Une grande proportion de Canadiens ne consomment pas les rations quotidiennes recommandées de fruits et légumes.

- L'âge peut influer sur l'absorption des nutriments.

\section{Recommandations}

En fonction des connaissances actuelles :

- Les médecins et les professionnels des soins de la vue devraient informer les adultes de 55 ans et plus des facteurs de risque liés à la DMLA. 
- Les médecins et les professionnels des soins de la vue devraient transmettre les stratégies de gestion des facteurs de risque pour la prévention de la DMLA aux patients à risque.

- Les médecins et les professionnels des soins de la vue devraient conseiller aux patients d'arrêter de fumer, de réduire au minimum l'exposition au soleil et d'adopter une saine alimentation

- L'étude Rotterdam Eye Study laisse entendre que l'apport alimentaire régulier de certaines vitamines antioxydantes et minéraux peut être profitable aux patients à risque DMLA précoce et à ceux qui ont des antécédents familiaux de DMLA. Quand le régime alimentaire ne peut satisfaire aux exigences quotidiennes en vitamines et minéraux pour réduire le risque de progression de la DMLA, les suppléments peuvent être une solution pour les patients à risque. Les médecins et les professionnels des soins de la vue devraient vérifier la consommation régulière de suppléments des patients avant de recommander d'autres suppléments contre la DMLA.

- Il faut poursuivre la recherche pour examiner les effets bénéfiques, les risques, et les doses idéales des suppléments de vitamines et de minéraux pour les patients à risque de DMLA.

\section{Remerciements}

Le présent manuscrit a été transcrit et mis en page par Medex Communications Inc., Oakville, Ontario, Canada. Medex Communications
Inc. n'est nullement affiliée aux auteurs du présent document.

Les auteurs ont reçu une subvention de recherche sans restriction offerte par Novartis Pharmaceuticals Canada Inc.

\section{Références}

1. Organisation mondiale de la Santé. Ampleur et causes des déficiences visuelles. Aidemémoire n 282; novembre 2004. Disponible : http:// www.who.int/mediacentre/factsheets/ fs282/fr/ (accessed 2007 Sept 14). (consulté le 14 septembre 2007).

2. Haddad S, Chen CA, Santangelo SL, Seddon JM. The genetics of agerelated macular degeneration: a review of progress to date. Surv Ophthalmol 2006;51:316-63.

3. Klein R, Klein BE, Linton KL. Prevalence of age-related maculopathy. The Beaver Dam Eye Study. Ophthalmology 1992;99:933-43.

4. Klein R, Klein BE, Knudtson MD, Meuer SM, Swift M, Gangnon RE. Fifteen-year cumulative incidence of age-related macular degeneration: the Beaver Dam Eye Study. Ophthalmology 2007;114:253-62.

5. Turcotte M, Schellenberg G. Un portrait des aînés an Canada 2006. Ottawa; Statistique Canada. № 89-519-XIE au catalogue. Février 2007.

6. Brown GC, Brown MM, Sharma S, Stein JD, Roth Z, Campanella J, et al. The burden of age-related macular degeneration: a value-based medicine analysis. Trans Am Ophthalmol Soc 2005;103:173-84.

7. Gupta OP, Brown GC, Brown MM. Age-related macular degeneration: the costs to society and the patient. Curr Opin Ophthalmol 2007;18:201-5.

8. Sippy B, Hinton D. Aging of retina and retinal pigment epithelium. In: Agerelated macular degeneration. Lim JI, ed. NY; Marcel Dekker Inc., 2002.

9. van Leeuwen R, Boekhoorn S, Vingerling JR, Witteman JC, Klaver CC, Hofman A, et al. Dietary intake of antioxidants and risk of agerelated macular degeneration. JAMA 2005;294:3101-7.
10. Suzuki M, Kamei M, Itabe H, Yoneda $\mathrm{K}$, Bando H, Kume N, et al. Oxidized phospholipids in the macula increase with age and in eyes with age-related macular degeneration. Mol Vis 2007;13:772-8.

11. Kamei M, Yoneda K, Kume N, Suzuki M, Itabe H, Matsuda K, et al. Scavenger receptors for oxidized lipoprotein in age-related macular degeneration. Invest Ophthalmol Vis Sci 2007;48:1801-7.

12. Age-Related Eye Disease Study Research Group. Risk factors associated with age-related macular degeneration. A case-control study in the age-related eye disease study: Age-Related Eye Disease Study Report Number 3. Ophthalmology 2000;107:2224-32.

13. Taylor HR, Munoz B, West S, Bressler NM. Visible light and risk of agerelated macular degeneration. Trans Am Ophthalmol Soc 1990;88:163-73.

14. Hyman L, Neborsky R. Risk factors for age-related macular degeneration: an update. Retina and vitreous disorders. Curr Opin Ophthalmol 2002;13:171-75.

15. Age-Related Eye Disease Study Research Group. A randomized, placebocontrolled, clinical trial of high-dose supplementation with vitamins $\mathrm{C}$ and $\mathrm{E}$, beta carotene, and zinc for age-related macular degeneration and vision loss: AREDS report no. 8. Arch Ophthalmol 2001;119:1417-36.

16. Age-Related Eye Disease Study Research Group. The relationship of dietary carotenoid and vitamin $\mathrm{A}, \mathrm{E}$, and $\mathrm{C}$ intake with age-related macular degeneration in a case-control study. AREDS Report No. 22. Arch Opbthalmol 2007;125:1225-32.

17. Tan JS, Wange JJ, Flood V, et al. Dietary antioxidants and the long-term incidence of ARMD: The Blue Mountain Eye Study. Ophthalmology 2007;115:334-41.

18. Moeller SM, Parekh N, Tinker L, et al. CAREDS Research Study Group. Associations between intermediate ARMD and lutein and zeaxanthin in the Carotenoids in Age-related Eye Disease Study (CAREDS): ancillary study of the Women's Health Initiative. Arch Ophthalmol 2006;124:1151-62.

19. Delcourt C, Carriere I, Delage M, et al., POLA Study Group. Plasma lutein and zeaxanthin and other carotenoids

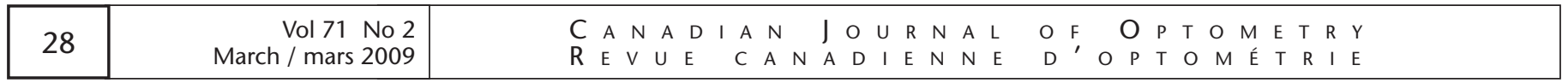


as modifiable risk factors for agerelated maculopathy and cataract: the POLA study. Invest Ophthalmol V is Sci 2006;47:2329-35.

20. Seddon JM, Ajani UA, Sperduto RD, Hiller R, Blair N, Burton TC, et al. Dietary carotenoids, vitamins $A$, $\mathrm{C}$, and $\mathrm{E}$, and advanced age-related macular degeneration. Eye Disease Case-Control Study Group. JAMA. 1994;272(18):1413-20. Erratum in: JAMA 1995;273(8):622.

21. Chong EW-T, Wong TY, Krels AJ, Simpson JA, Guymer RH. Dietary antioxidants and primary prevention of age-related macular degeneration: systematic review and meta-analysis. Available: http://www.bmj.com/ cgi/content/short/335/7623/755 (published 8 October 2007). Ref \# doi:10.1136/bmj.39350.500428.47.

22. Seddon JM, Cote J, Page WF, Aggen SH, Neale MC. The US twin study of agerelated macular degeneration: relative roles of genetic and environmental influences. Arch Ophthalmol. 2005;123(3):321-7.

23. Haines JL, Spencer KM, PericakVance MA. Bringing the genetics of

\section{OPTOMETRIST}

\section{Required Part Time}

\author{
for a busy \\ Medical clinic \\ in Etobicoke
}

\section{3-4 hours a week}
Call: Dr. Mansour at 416-660-2797

macular degeneration into focus. PNAS 2007;104:16725-26.

24. Hyman LG, Lilienfeld AM, Ferris FL 3rd, Fine SL. Senile macular degeneration: a case-control study. Am J Epidemiol 1983;118:213-27.

25. Frank N, Puklin JE, Stock C, Canter LA. Race, iris color, and age-related macular degeneration. Trans Am Ophthalmol Soc 2000;98:109-15.

26. Clemons TE, Milton RC, Klein R, Seddon JM, Ferris FL 3rd; Age-Related Eye Disease Study Research Group. Risk factors for the incidence of advanced age-related macular degeneration in the Age-Related Eye Disease Study (AREDS). AREDS report no. 19. Ophthalmology 2005;112:533-9.

27. Santé Canada. Guide alimentaire canadien 2007. Nombre de portions du Guide alimentaire canadien recommandé chaque jour. Disponible : http://www. hc-sc.gc.ca/fn-an/food-guide-ailment/ order-commander/eating_well_ bien_manger_fra.html. (Consulté le 13 sept. 2007.)

28. Statistique Canada. Tableau 1050449. Consommation de fruits et légumes, selon le groupe d'âge et le sexe, population

\author{
à domicile de 12 ans et plus, Canada, \\ provinces, territoires et certaines régions \\ sociosanitaires (limites de juin 2005), aux \\ 2 ans (175392 séries). Disponible : http:/ / \\ cansim2.statcan.ca/cgi-win/cnsmcgi. \\ exe?Lang $=\mathrm{F} \&$ RootDir $=\mathrm{CII} / \&$ Res \\ ult Template $=\mathrm{CII} / \mathrm{CII} \_\&$ Array_ \\ Pick $=1 \&$ ArrayId $=1050449$. (Consulté \\ le 28 mai 2007.)
}

Mots clés : dégénérescence maculaire liée à l'âge, prévention, maladie oculaire dégénérative, suppléments d'antioxydants, suppléments oculaires, facteurs de risque, consensus, ligne directrice

Sohel Somani* MD, Ann Hoskin-Mott ${ }^{\dagger}$ MD, Adit Mishra MD, Alain Bois ${ }^{\ddagger}$ OD, Brian H. Book ${ }^{\S}$ OD, Mark Chute ${ }^{\Omega}$ OD, Ronald Gaucher ${ }^{¥}$ OD, Barry Winter ${ }^{\curvearrowright}$ OD.

De *Toronto, Ont., †Halifax, N.-É., ${ }^{\ddagger}$ Longueuil, Québec., 'Winnipeg, Man., ${ }^{\Omega}$ Elmsdale Vision Centre, Elmsdale, N.-É., ${ }^{\sharp}$ Regina, Sask., ${ }^{2}$ Toronto, Ont.

\section{Are you doing all you can to maximize the revenue potential of your practice?}

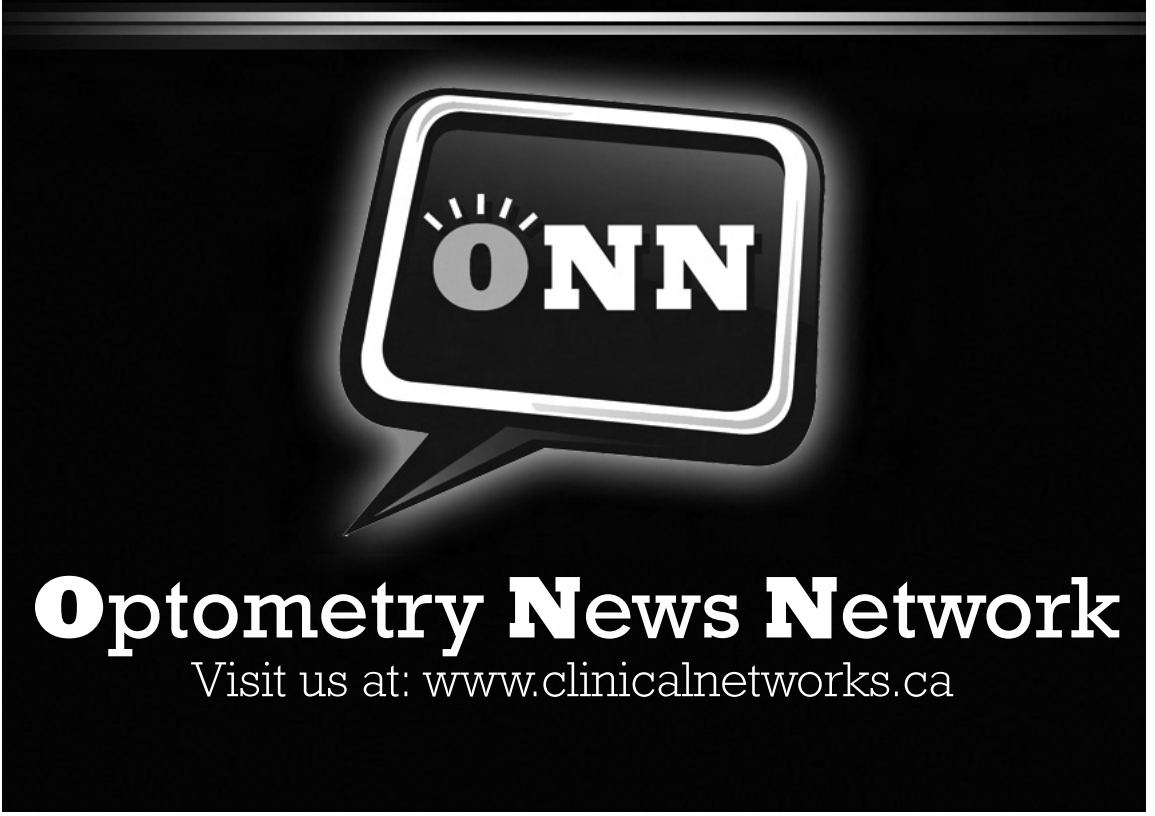


Tableau 1 : Nombre estimé de Canadiens affectés par la DMLA

\begin{tabular}{|l|c|c|}
\hline Âge (années) & $\begin{array}{l}\text { Pourcentage (\%) } \\
\text { de personnes } \\
\text { souffrant de DMLA }\end{array}$ & $\begin{array}{l}\text { Canadiens } \\
\text { souffrant de } \\
\text { DMLA* }^{*}\end{array}$ \\
\hline $43-53$ & 8.5 & 467,500 \\
$55-64$ & 14.4 & 529,126 \\
\hline $65-74$ & 19.4 & 440,768 \\
$\geq 75$ & 36.8 & 748,512 \\
\hline Total estimé & & $2,185,906$ \\
\hline
\end{tabular}

* Extrapolation à partir des données de la Beaver Dam Eye Study 1988-19903 Statistiques démographiques pour les groupes d'âge de moins de 65 ans: Recensement de 2006 de Statistique Canada; pour les groupes d'âge de plus de 65 ans, prévisions de Statistique Canada (croissance moyenne), 2005
Tableau 2 - Pourcentage de Canadiens

en 2005 qui ont consommé moins

de cinq portions/jour de fruits et légumes

\begin{tabular}{|l|c|}
\hline Âge (années) & Pourcentage(\%) \\
\hline $45-54$ & 56.8 \\
$55-64$ & 52.3 \\
$65-74$ & 48.5 \\
$\geq 75$ & 39.4 \\
\hline
\end{tabular}

Statistique Canada, tableau 105-044928

Figure 1 - Pathogénèse de la DMLA

\section{Angiogrammes à la fluorescéine}

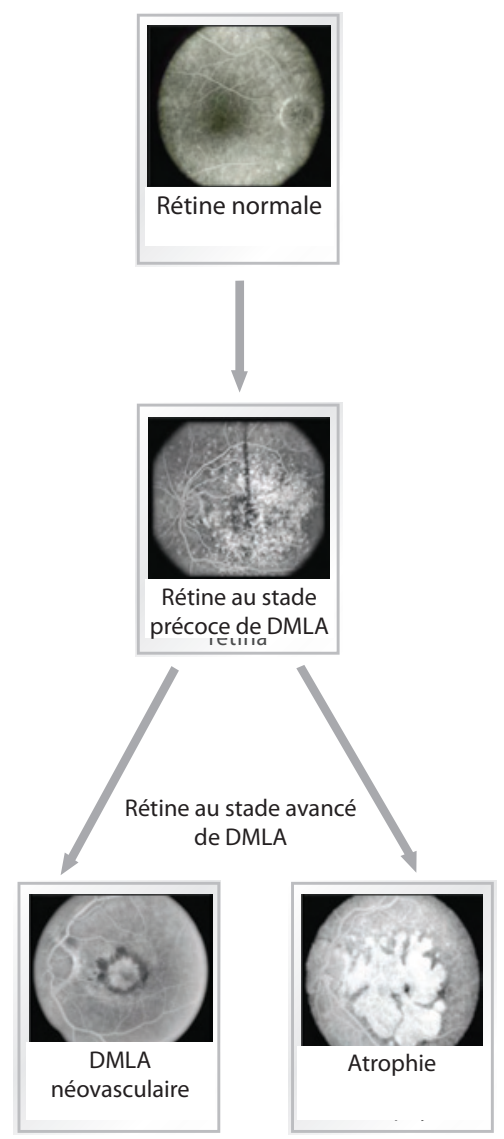

NVC $=$ Néovascularisation choroïdienne

\section{Stress oxydatif}

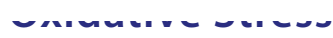

Activation du complément

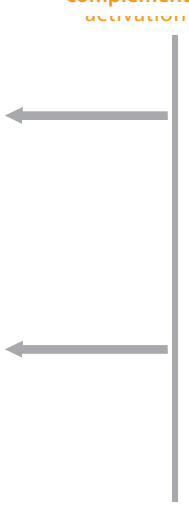

Inflammation

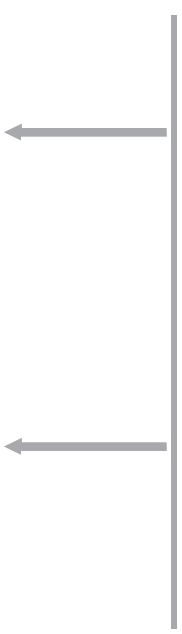

